Archives

$42 \mid 2008$

Circulations et frontières

\title{
Libre circulation des marchandises et contrôle de la mobilité des hommes dans les territoires de la monarchie des Habsbourg
}

Contradiction ou complémentarité?

\section{Andrea Komlosy}

\section{(2) OpenEdition \\ Journals}

Édition électronique

URL : http://journals.openedition.org/ccrh/3436

DOI : $10.4000 /$ ccrh.3436

ISSN : $1760-7906$

Éditeur

Centre de recherches historiques - EHESS

Édition imprimée

Date de publication : 25 avril 2008

Pagination : 117-130

ISSN : 0990-9141

Référence électronique

Andrea Komlosy, «Libre circulation des marchandises et contrôle de la mobilité des hommes dans les territoires de la monarchie des Habsbourg », Les Cahiers du Centre de Recherches Historiques [En ligne], 42 | 2008, mis en ligne le 29 novembre 2011, consulté le 20 avril 2019. URL : http:// journals.openedition.org/ccrh/3436 ; DOI : 10.4000/ccrh.3436

Ce document a été généré automatiquement le 20 avril 2019.

Article L.111-1 du Code de la propriété intellectuelle. 


\section{Libre circulation des marchandises et contrôle de la mobilité des hommes dans les territoires de la monarchie des Habsbourg}

Contradiction ou complémentarité?

Andrea Komlosy

\section{Les territoires et la période en question} les possessions espagnoles, qui furent séparées au XVI ${ }^{\mathrm{e}}$ siècle) ;

3 - des acquisitions du XVI ${ }^{\mathrm{e}}$ siècle, notamment de la Bohême, de la Moravie et de la Silésie (à partir de 1526) ;

4 - des expansions du XVIII ${ }^{\mathrm{e}}$ siècle, notamment de la Galicie (acquise en 1772 et 1795 par le partage de la Pologne), de la Bucovine (acquise en 1775 et appartenant à la Galicie entre 1775 et 1850) et des côtes dalmatiennes (acquises en 1797), lesquelles faisaient partie des « Pays autrichiens », administrés par Vienne. Le pluriel indique qu'il s'agit de l'ensemble des territoires sous le pouvoir Habsbourg; au singulier, "pays » signifie province. Le terme « état » est par contre réservé au gouvernement et à l'administration centrale des pays du complexe Habsbourg ;

5 - La Hongrie avait un roi Habsbourg et faisait partie des possessions des Habsbourgs ; ici, elle est pourtant considérée comme un État en tant que tel, avec lequel l'Autriche entretenait des relations internationales ${ }^{1}$;

6 - Les Pays-Bas autrichiens (1714-1797), la Lombardie (1714-1797, 1815-1859) et la Vénétie (1797-1805, 1815-1866) ne firent partie de l'Autriche que temporairement. Ils n'étaient ni 
soumis à l'administration centrale ni inclus dans la formation d'un marché commun et ne sont donc pas inclus dans les considérations suivantes.

7 La période en discussion commence par les grandes réformes d'État portant sur le système administratif et fiscal, les droits seigneuriaux, la circulation des marchandises ainsi que sur la liberté et la mobilité des hommes. Ces réformes furent introduites en Autriche à partir de 1750. Notre période se termine avec l'effondrement de l'Empire en 1914. Cet article est centré sur les frontières et les circulations à l'intérieur des «Pays autrichiens», lesquelles représentaient plus de $95 \%$ des mouvements commerciaux et des déplacements personnels. Il confronte deux évolutions qui paraissent contradictoires. Pendant la période en discussion, la circulation des marchandises fut libéralisée, tandis que la circulation des hommes fit l'objet d'un contrôle de régulation. La question qui se pose est de savoir comment l'un était lié à l'autre et quel était le rôle des frontières dans la formation de l'administration centrale de l'état et du marché national. Les frontières servaient-elles de frein à la circulation ou bien au contraire d'instrument d'intégration ${ }^{2}$ ?

\section{La libéralisation du commerce}

$8 \mathrm{Au} \mathrm{XVIII}$ siècle, la monarchie Habsbourg se caractérisait par l'homogénéisation du marché (national), marquée par :

9 - l'abolition des douanes internes (Binnenzölle) entre les pays (Länder) ${ }^{3}$. L'unification du marché interne affecta la plupart des anciens pays autrichiens et les pays bohémiens en 1775, puis la Galicie en 1784, et le Tyrol, la Lombardie et la Vénétie en 1825-1826. La Hongrie ne fût inclue dans le marché commun qu'en 1851, la Dalmatie en 1879;

10 - la transformation des péages (Mauten) ${ }^{4}$. Les péages perdirent de l'importance et devinrent une rémunération pour l'usage d'une route, d'un pont, d'un bac etc. Le nombre des péages seigneuriaux ou municipaux (dites privés par l'état) fut réduit à partir du XVII e siècle, puis soumis à la législation (1755) et à l'administration étatique (1788). En même temps, l'État créa de nouveaux péages, consacrés à la construction de routes, qui étaient levés par des fermiers.

11 En outre, il était d'usage de lever des taxes ou traites sur la circulation des marchandises par droit municipal, ce qui était souvent le privilège des corporations. Ils obligeaient les marchands non seulement à utiliser des routes prescrites (passant par les douanes), mais aussi à s'arrêter dans les villes situées sur la route, à y offrir leurs produits et à payer tous les frais liés à la vente. Ces droits aussi furent abolis après $1775^{5}$.

\section{La régulation du passage}

12 Contrairement à l'ouverture du marché, le passage des hommes devint l'objet du contrôle étatique, représentant désormais un processus de régulation.

13 Jusqu'à 1750 , le droit de passage dépendait du statut social ou professionnel des personnes. Les nobles et le clergé jouissaient du libre passage ; les marchands et artisans effectuaient le passage avec l'aide de leurs réseaux professionnels; les paysans et autres personnes soumises à l'autorité seigneuriale avaient besoin d'une autorisation spéciale, la majorité étant liée au domaine ; quant aux personnes sans terre, professions itinérantes, vagabonds, mendiants etc., ils voyageaient sans autorisation. 
14 À partir de 1750 - date d'une réforme majeure de l'État - le passage fut réglé par la loi : en dehors des nobles et des personnes non soumises au service militaire, tout le monde avait besoin d'une permission, c'est-à-dire d'un passeport ou d'autres documents pour franchir les frontières de son département de séjour (Kreis). En allemand de l'époque, un voyage à travers les frontières du département était désigné par le terme Außerkreisgehen; le contraire, qui signifie rester dans les limites des son département, Imkreisgehen, est encore utilisé de nos jours au sens large pour désigner une situation de laquelle on est prisonnier.

$15 \mathrm{Au}$ début, les règles de passage différaient selon l'origine sociale - une date importante fut l'abolition du servage en 1781, qui avait été en vigueur dans quelques provinces (pays/ Länder) jusque-là. On peut observer une homogénéisation progressive des régulations, qui signifie aussi la monopolisation par l'État.

Jusqu'en 1820, les anciennes institutions, par exemple les corporations et confréries, avaient le droit de fournir à leurs membres des documents de passage. Non seulement ces organisations perdirent certains privilèges, mais l'artisan itinérant devint l'objet d'une surveillance rigoureuse, ce qui entrava sa mobilité traditionnelle?

Les règles de contrôle du passage furent de plus en plus générales; elles étaient fondées sur les nouveaux découpages administratifs, que le pouvoir central érigea à l'intérieur de l'État. Ce processus peut être observé à partir de 1750, quand le pouvoir central essaya de supprimer le pouvoir seigneurial à tous les niveaux juridiques et administratifs, notamment au niveau des domaines ${ }^{10}$.

En Autriche, la réforme étatique n'était pas initiée par une révolution comme en France, elle fut réalisée par le pouvoir impérial et sa bureaucratie, et fondée sur un compromis entre la noblesse et le pouvoir impérial. La noblesse conserva le droit d'autogestion des domaines, mais en même temps, elle fut transformée pour devenir la première instance de l'administration centrale, responsable de la collecte des impôts, de la conscription et de l'autorisation du passage.

19 Au même moment, l'État créa de nouvelles unités administratives qui ne dépendaient pas du pouvoir noble - ni de ses représentations, par exemple les États Généraux (Stände) et les diètes (Landtage) dans les pays - et qui contrôlaient et surveillaient l'administration de la première instance (seigneuriale) - une situation qui reflète les intérêts contradictoires en jeu. Sur le plan régional, le niveau administratif, qui exprimait l'intérêt du pouvoir central, était le département (Kreis). Jusqu'au XVII ${ }^{e}$ siècle, le Kreis (cercle) était une unité administrative revendiquée par le pouvoir central aussi bien que par l'aristocratie provinciale. À partir de 1748 , ces anciennes unités perdirent leur autonomie administrative et furent transformées en circonscription étatique, contrôlée par le gouvernement central ${ }^{11}$. En rapport avec la réforme administrative autrichienne, la transformation des circonscriptions régionales correspond à l'introduction du département en France à partir de 1789. Au début, les administrations départementales ( Kreisämter) furent chargées de la conscription et de la surveillance de seigneuries. Au fur et à mesure, elles devinrent responsables de tous les domaines de l'administration publique dans leurs frontières, constituant ainsi le lien immédiat entre l'État et les sujets, et transformant ceux-ci par-là même en citoyens.

20 Sur le plan des provinces (pays/Länder), l'État se servit des gouvernements provinciaux, qui perdirent leur ancienne autonomie. Les institutions des États Généraux, les diètes et leurs administrations devinrent donc un instrument de l'administration centrale ${ }^{12}$. Ici, je 
m'intéresse particulièrement aux unités territoriales crées et contrôlées par l'administration centrale, parce que ces unités-là fournissaient l'instrument de contrôle des passages : les frontières.

21 L'espace étatique fut donc territorialisé, en même temps, on le découpa en unités régionales et locales selon un principe hiérarchique, lequel définit le champ de compétence de chaque unité. Ainsi, il devint possible d'identifier chaque personne à l'aide de son appartenance à un territoire précis, de régler les droits de séjour et les droits sociaux et d'établir des règles pour quitter le territoire ou pour passer ses frontières.

Les règles de passage étaient contradictoires: D'un côté, elles encourageaient la circulation des hommes, puisqu'elles réduisaient les droits des domaines de limiter le mouvement de leurs sujets. D'un autre côté, elles servaient d'instrument de contrôle et de sélection des voyageurs et de la main d'œuvre migratoire, reflétant des intérêts divergents (pouvoir central - pouvoir régional et local ; état - cercles économiques, ville/ campagne, agriculteurs - industriels etc.).

\section{Moyens de contrôle du passage}

23 Nous pouvons identifier deux moyens principaux de contrôle du passage, qui correspondent aux différentes périodes :

24 - 1750-1850 : La délivrance de passeports ;

$25-1850-1900$ : Le droit de domicile (Heimatrecht) et l'expulsion (Schub, Abschiebung) dans la commune de domicile.

\section{Passeports}

26 À part quelques exceptions, un passeport était obligatoire pour chaque personne qui quittait son département, soit pour un court voyage soit pour un séjour plus long ${ }^{13}$. En délivrant le passeport, le pouvoir central obtenait la preuve de chaque déplacement et la possibilité de l'autoriser ou pas. L'autorité habilitée à délivrer les passeports variait selon les personnes et les destinations. Les nobles et le clergé n'avaient besoin d'un passeport que pour les voyages dans un pays étranger; leurs voyages étaient pourtant mal vus, parce qu'il ne servaient pas des buts considérés comme utiles. Pour supprimer les voyages exclusivement réservés à la noblesse (adelige Kavalierstouren), les jeunes aristocrates ne furent autorisés passer les frontières qu'à l'âge de 28 ans. D'autres régulations spéciales existaient pour les étudiants, surtout pendant les Guerres napoléoniennes, quand tout contact intellectuel à travers les frontières était suspect. À cette période, nobles, clergé et étudiants avaient besoin d'une autorisation des autorités suprêmes pour obtenir un passeport. Pour tous les autres groupes de la population, qui avaient besoin d'un passeport pour les voyages à l'intérieur de l'État, la compétence administrative varia selon les destinations: les administrations seigneuriales étaient responsables des passeports aussi pour un voyage dans les limites du pays, les administrations départementales étaient responsables pour un voyage dans un autre pays, pourvu qu'il fasse parti de l'ensemble des «pays conscrits " (konskribierte Länder), où la conscription militaire était sous contrôle d'état. Tous les autres voyages étaient administrés par les autorités provinciales ou centrales. En plus, tout homme soumis au recrutement avait 
besoin d'une autorisation spéciale des autorités militaires pour obtenir un passeport, ce qui en général n'était le cas qu'à la fin du service militaire ${ }^{14}$. l'éligibilité à une fonction communale etc. Le droit de domicile, qui date du XVI 
représente une manière de rattacher une personne à une certaine commune - un ancrage spatial des hommes, qui permet aux autorités politiques d'envoyer ceux qui ne sont pas domiciliés chez eux dans leur commune de rattachement (erzwungene Abschiebungexpulsion par force) ${ }^{17}$. Pendant les XVII et $\mathrm{XVIII}^{\mathrm{e}}$ siècles, la législation avait prévu la possibilité pour une personne d'obtenir le droit de domicile sur son lieu de résidence après dix ans de séjour permanent.

En 1863, cette pratique fut abandonnée pour une nouvelle loi de domicile, qui transformait ainsi le droit de domicile en moyen de contrôle des migrations et des lieux de résidence. Le séjour permanent ne suffit plus pour obtenir le droit de domicile ; celuici n'était plus concédé que si le demandeur était bienvenudans sa nouvelle commune; sinon, il demeurait un habitant sans y avoir le droit de domicile ${ }^{18}$.

Pendant les années de migration la plus intense et de croissance des grands centres urbains et industriels dans la deuxième moitié du XIXe siècle, les émigrés étaient donc privés de la possibilité d'obtenir le droit de domicile dans leur nouvelle résidence - ce qui les rendait citoyens de deuxième catégorie, menacés par l'expulsion de force dans leur commune de domicile (Heimatgemeinde, rechtliche Heimat - domicile de droit) en cas de pauvreté, chômage ou perte d'assistance familiale.

Comme les recensements (de la population), qui étaient effectués tous les dix ans à partir de 1857, enregistraient la domiciliation, on peut étudier la façon dont se développèrent les relations entre les " autochtones ", (c'est-à-dire les " domiciliés ») et les "étrangers " (c'est-à-dire les personnes sans droit de domicile sur place) dans une certaine unité territoriale. Au fur et à mesure que le nombre des immigrants dans les centres urbains et industriels augmentait, le pourcentage des habitants domiciliés tomba. Le résultat ne dit rien sur le mouvement d'immigration et d'émigration par période d'observation (qui est documenté par ailleurs). Mais il montre le pourcentage de personnes qui n'étaient pas domiciliées dans leur commune de séjour au jour du recensement, un nombre qui augmentait au fur et à mesure que les gens immigraient sans être domiciliés sur leur nouveau lieu de résidence ${ }^{19}$.

Malgré les distorsionsdu résultat du fait que l'épouse avait le droit de domicile dans la commune où était domicilié son mari, les chiffres montrent bien combien résidence et domiciliation divergent.

Tableau : Personnes domiciliées à Vienne et à Prague, 1857-1900 


\begin{tabular}{|c|c|c|}
\hline & Vienne & Prague \\
\hline \hline 1857 & 60 & 50 \\
\hline 1869 & 45 & 36 \\
\hline 1880 & 35 & 30 \\
\hline 1890 & 35 & 30 \\
\hline 1900 & 38 & 21 \\
\hline
\end{tabular}

Source : Österreichische Statistik, Volkszählungen 1857-1900 ; Andrea Komlosy, Grenze, p. 464 «rapatriement $»^{20}$. En effet tous les gens qui n'étaient pas domiciliés dans leur lieu de séjour étaient également plus enclins à accepter des conditions de vie et de travail plus difficiles que leurs compatriotes domiciliés. Ce qui fit de la loi de domicile non seulement un instrument de contrôle, mais aussi de coercition sociale. Elle resta en vigueur jusqu'en 1896, apogée de la croissance des grandes villes, moment où il devint nécessaire de légaliser l'immigration qui avait eu lieu. Donc, le nombre des domiciliés augmenta à Vienne à partir de 1900. En même temps, les municipalités commencèrent à établir des institutions d'assistance sociale ${ }^{21}$. À Prague, le nombre des domiciliés n'augmenta pas encore, car les limites de la commune n'avaient pas été élargies, comme ce fut le cas à Vienne, qui connut plusieurs vagues d'élargissement. Ainsi, les habitants du (centre) ville de Prague restèrent "étrangers", même s'ils étaient originaires des faubourgs constituant encore des communes particulières.

\section{Les rapports aux circulations et aux frontières}

L'abolition des douanes et péages entre les provinces (pays) de l'Autriche à partir de 1775 facilita la circulation des marchandises. Pourtant, l'homogénéisation du marché coïncida avec une polarisation qui accrut les différences entre les régions (les disparités régionales), créant " centres » et " périphéries », divisés par des frontières économiques.

Cette polarisation causa une nouvelle qualité de migration, caractérisée par des distances plus longues. Néanmoins, c'était toujours une migration qui servait de revenu supplémentaire à la région d'origine. C'est seulement à la fin du XIXe siècle que les migrations circulaires donnèrent lieu à une émigration définitive - et même celle-là restait étroitement liée avec la région d'origine des émigrés ${ }^{22}$.

La migration la plus intense s'observait dans les régions centrales et économiquement développées du pays, où "centres » et "périphéries " existaient sur un plan microrégional, notamment à l'intérieur de et entre les provinces de la Basse et de la Haute Autriche, la Styrie, la Bohême et la Moravie. En revanche, la contribution des provinces agraires à l'est et au sud-est - notamment la Galicie, la Bucovine et la Dalmatie - à la migration interne, fut plus basse. Quand, à la fin du XIXe siècle, dans ces pays aussi, il 
devint nécessaire, pour la plus grande partie de la population, de chercher du travail supplémentaire à l'extérieur de la région, la migration se tourna vers l'Allemagne et les États-Unis ${ }^{23}$. Ainsi, la migration entre les régions en voie d'industrialisation et les provinces agraires à l'est et au sud-est était moins dense, ce qui confirme la thèse que la migration ne résulte pas d'une différence absolue, mais des interactions établies qui étaient les plus développées dans les régions du cœur du pays ${ }^{24}$.

Ici tout mouvement, s'il faisait franchir les frontières communales ou les frontières internationales, est considéré comme circulation. La régulation des déplacements avait lieu à plusieurs échelles administratives, ce qui permettait d'établir une distinction entre «l'autochtone» et «l'étranger ».Pour les voyages, la distinction fondamentale se présentait entre le département résidentiel, dans lequel un déplacement n'exigeait pas d'autorisation administrative, et chaque circulation au-delà de cet espace, où le voyageur était qualifié d'étranger. Cette distinction perdit sa signification avec l'abolition des passeports internes en 1857.

Quant au droit de domicile, la distinction fondamentale se présentait entre la région terroir (" Heimat »), qui était définie par les confins communaux, et la région étrangère (" Fremde ») : là où une personne ne disposait pas du droit de domicile et donc était qualifiée d'étranger ${ }^{25}$. Dans ces deux cas, la différence entre les hommes ne s'exprimait pas par la nationalité, mais par les droits de passage et par les droits communaux.

La Constitution de l'État (Staatsgrundgesetz 1867) a (re)confirmé la notion de nationalité, que nous connaissons aujourd'hui : celle qui est réservée aux citoyens d'un État. À partir de ce moment-là, l'Autriche était caractérisée par deux aspects de la citoyenneté :

- une garantie par la constitution, qui exprimait les droits politiques fondamentaux des citoyens et, par cela, le libre choix de résidence dans le pays entier ;

- une autre garantie par le droit de domicile, qui exprimait les droits communaux. Ces derniers étaient valides dans les limites de la commune de droit. En d'autres termes, si quelqu'un avait besoin d'assistance sociale, il était rattaché à une seule commune; en cas de perte de travail ou de soutien familial, on l'y envoyait par « déportation ».

En ce qui concerne les relations entre la circulation des marchandises et la circulation des hommes, on peut donc résumer :l'abolition des douanes et péages internes représenta une dérégulation. Elle correspondait au règlement du passage, qui, par contre, représenta une régulation. La libre circulation des marchandises contribuait à la mobilité (des voyageurs, des migrants travailleurs...), créant ainsi la nécessité de régler les flux migratoires. Il ne s'agissait donc pas d'une contradiction, mais d'une complémentarité.

\section{Frontières : barrages ou liaisons?}

47 Tout déplacement au-delà des limites de la commune résidentielle faisait franchir des frontières. Tout d'abord, on pense aux frontières politiques et administratives. Par rapport aux déplacements à l'intérieur de la monarchie autrichienne, les nouvelles frontières politiques et administratives, créées dans la deuxième moitié de XVIII ${ }^{e}$ siècle, constituaient un nouveau réseau caractérisé par des espaces nettement séparés, entourés de frontières linéaires, représentant un ordre hiérarchique, qui permettait de rattacher chaque personne à son terroir et de régler par loi les droits de séjour et de passage. 

plus vagues et plus relatives. L'homogénéisation du marché interne les a accentuées, parce que la compétition contribuait à la croissance des disparités régionales. Et l'homogénéisation les a flexibilisées, parce que les relations interrégionales subissaient une transformation permanente. L'attachement des hommes aux espaces économiques est également changeant et dépend du contexte, parce que la migration circulaire lie les régions d'origine aux destinations de la migration, contribuant ainsi aux identités transversales. la position qu'une région occupait sur le marché national. Chaque changement de ces relations influençait la décision des hommes de rester dans leur région terroir ou bien de chercher du travail ailleurs. Il y a ainsi une relation étroite entre les frontières économiques et les frontières administratives, caractérisée par une forte tension. Les frontières économiques encouragent ou nécessitent les migrations; les frontières administratives fournissent le cadre spatial pour découper l'espace migratoire en territoires précis et contrôlables. frontière ethnolinguistique entre les peuples de l'Empire austro-hongrois, unis par les liens de la dynastie régnante. Elles aussi se recoupaient avec les frontières administratives, ce qui donna aux mouvements nationaux l'idée de faire coïncider les deux types de frontières. Il est établi que les tensions ethnolinguistiques contribuèrent à l'échec de l'Empire. En ce qui concerne l'autorisation et le contrôle de la circulation, elles ne jouèrent aucun rôle décisif. A l'époque des passeports internes (1748-1857), nous n'avons pas d'indication sur l'influence de l'ethnie ou de la langue sur la décision des bureaux de délivrer un passeport ou non. Quant aux conditions pour obtenir le droit de domicile, l'ethnie et la langue ne comptaient pas non plus. La distinction entre " autochtone» et "étranger" ne se faisait pas selon les groupes linguistiques; elle dépendait simplement du droit de domicile, et n'avait aucun lien avec l'appartenance ethnique. Une personne germanophone d'un village de Basse-Autriche à 25 kilomètres de Vienne avait le même statut "d'étranger » à Vienne que son compatriote tchèque, slovène ou polonais. Pourtant, il y avait de relations étroites entres les personnes de la même langue et de la même ethnie pendant les migrations aussi bien que dans les lieux de leur nouvelle résidence.

51 En divisant le territoire et en rattachant les hommes à ces subdivisions politiques, les frontières séparent. Cette observation s'applique aux frontières linéaires ainsi qu'aux frontières économiques définies par différents niveaux de développement et de prospérité selon les régions.

Dans le même temps les frontières unifient non seulement parce qu'elles sont en permanence illégalement franchies, mais surtout, parce qu'elles constituent des règles pour entrer dans et quitter les espaces administratifs, ce qui, en fait, les relie l'un à l'autre. D'où ma thèse : les frontières séparent afin d'unifier.

53 Le rattachement des hommes à des espaces administratifs précis présentait plusieurs intérêts. D'abord, il permettait aux administrations d'obtenir un tableau synoptique de la population, de ses lieux de résidence, de son domicile et de ses mouvements, ce qui était dans l'intérêt de l'administration centrale d'État. En même temps, ce rattachement administratif permettait de régler la circulation entre des régions séparées par des 
inégalités socio-économiques. Cette régulation était aussi bien dans l'intérêt de l'État que dans celui des entrepreneurs qui avaient besoin d'une main d'œuvre spécifique dans des lieux précis, ce qui exigeait une sélection.

Si la question de sélection est posée du point de vue des régions de départ, elle porte sur le droit de partir : qui avait droit aux documents de passage ? Si elle est posée du point de vue des destinations d'arrivée d'un déplacement, elle porte sur les possibilités de venir. En même temps, la question se pose de savoir qui - parmi ceux qui sont venus - avait le droit de rester, c'est-à-dire, jouissait du droit de domicile.

Ainsi le réseau des frontières économiques (séparant "centres» et "périphéries») recoupe le réseau constitué par les frontières administratives : c'est par la séparation que la liaison devient enfin possible. Le rattachement administratif est uniforme, il est fondé sur la naissance, le mariage, le patrimoine. En revanche, l'attachement aux régions économiques est dynamique, parce que les hommes se déplacent selon les opportunités sociales et économiques, qui sont inégalement distribuées selon les régions. Dans la deuxième moitié du XIXe siècle, quand la migration vers les centres urbains et industriels a été la plus intense, résidence (faktischer Aufenthalt) et domiciliation (rechtliche Heimatzugehörigkeit) étaient en contradiction flagrante, ce qui a rendu les migrants extrêmement vulnérables : ils étaient devenus des « étrangers » dans leur propre pays.

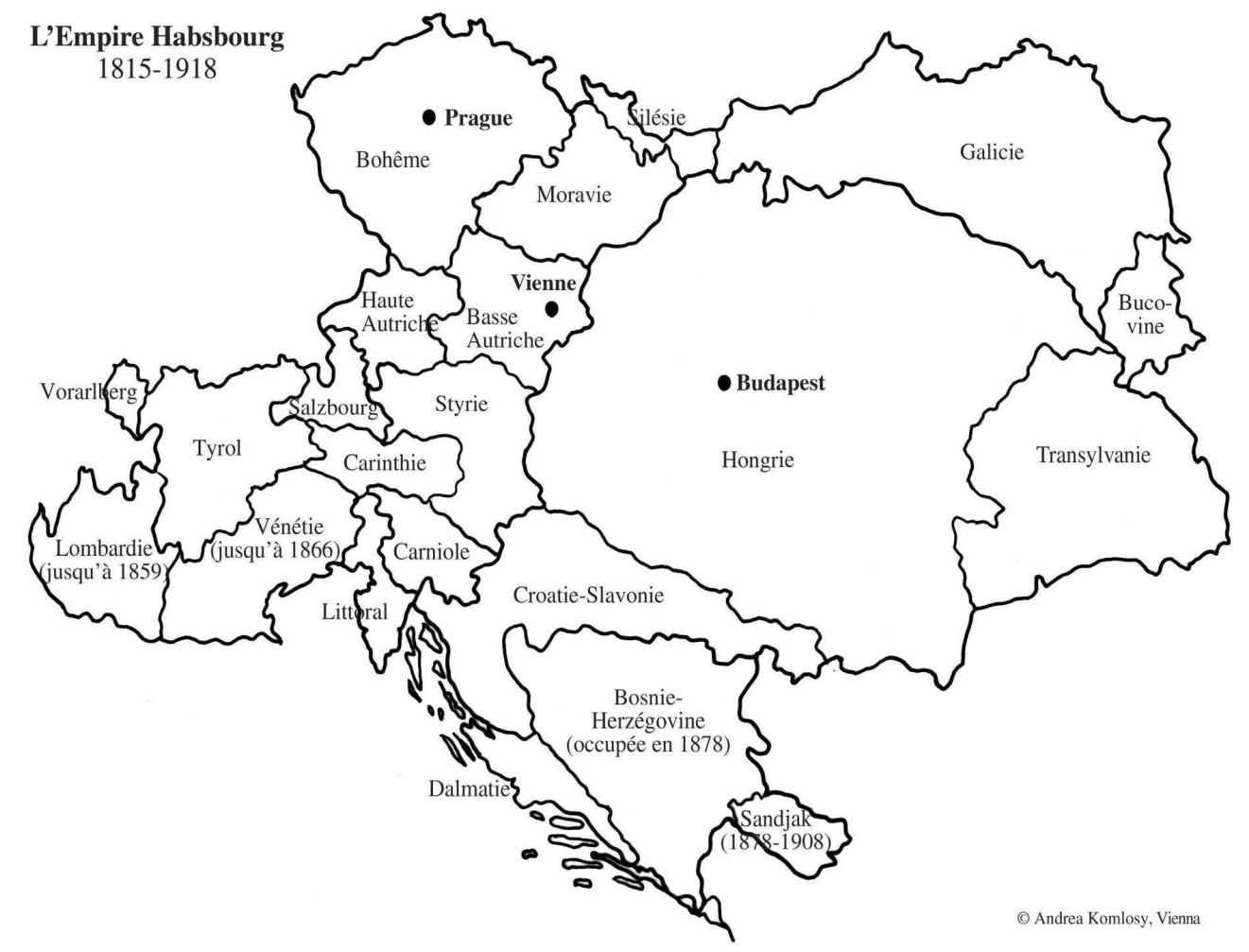




\section{NOTES}

1. Les relations avec la Hongrie furent soumises à plusieurs changements. Jusqu'en 1699-1718 la plus grande partie de celle-ci était sous domination turque, ce qui explique que les prétentions des Habsbourg ne furent réalisées qu'après le recul de l'Empire ottoman. Néanmoins l'aristocratie hongroise défendit son autonomie féodale contre les ambitions centralisatrices de Vienne, ce qui aboutit à une union personnelle entre les deux États en 1867, laquelle ne soumettait à l'administration commune que l'armée, la politique étrangère et le commerce extérieur. En ce qui concerne la circulation des marchandises, la Hongrie fut inclue dans le marché commun en 1851 ; en ce qui concerne la circulation des hommes, les deux entités furent considérées comme des états indépendants.

2. L'article est fondé sur l'habilitation de l'auteur à l'Institut d'Histoire économique et sociale à l'Université de Vienne : Andrea Komlosy, Grenze und ungleiche regionale Entwicklung. Binnenmarkt und Migration in der Habsburgermonarchie, Wien, Promedia, 2003.

3. Adolf Beer, «'Die Zollpolitik und die Schaffung eines einheitlichen Zollgebietes unter Maria Theresia ${ }^{\circ} »$, in Mittheilungen des Instituts für oesterreichische Geschichtsforschung, XIV, Innsbruck, 1893, p. 237-326 ; Andrea Komlosy, Grenze [...], op. cit., p. 134.

4. Benjamin Bowman, "Das Mautwesen des 18. Jahrhunderts im heutigen Niederösterreich", thèse non publiée, Wien, 1950, p. 126-128.

5. Benjamin Bowman, « Das Mautwesen [...] », op. cit., p. 99 ; Andrea Komlosy, Grenze [...], op. cit., p. 49.

6. Edith Saurer, Straße, Schmuggel, Lottospiel. Materielle Kultur und Staat in Niederösterreich, Böhmen und Lombardo-Venetien im frühen 19. Jahrhundert, Göttingen, Vandenhoeck \& Ruprecht, 1989, p. 189.

7. Adolf Beer, « Die Zollpolitik[...]», op. cit., p. 243-268.

8. Andrea Komlosy, "Ökonomische Grenzen », in Waltraud Heindl et Edith Saurer (ed.), Grenze und Staat. Passwesen, Staatsbürgerschaft, Heimatrecht und Fremdengesetzgebung in der österreichischen Monarchie (1750-1867), Wien-Köln-Weimar, Bôhlau, 2000, p. 823-827 ; A. Komlosy, Grenze [...], op. cit, p. 40-44.

9. Hannelore Burger, «Passwesen und Staatsbürgerschaft ${ }^{\circ} »$, in Waltraud Heindl et Edith Saurer (ed.), op. cit., p. 64 ; Zdenka Stoklásková, «Fremdsein in Böhmen und Mähren », in Waltraud Heindl/Edith Saurer (ed.), op. cit., p. 680.

10. Andrea Komlosy, Grenze [...], op. cit., p. 60-73.

11. Ignaz Beidtel, Geschichte der österreichischen Staatsverwaltung 1740-1848, 1, Innsbruck, 1896, p. 30-34; A. Komlosy, Grenze [...], op. cit., p. 62; Ernst von Plener, «ํine Kreisordnung für Böhmen», in Zeitschrift für Volkswirtschaft, Socialpolitik und Verwaltung, 3, Wien, 1899; Franz Stundner, «Die Kreisämter als Vorläufer der politischen Behörden I, Instanz (1748-1848)», in Johannes Gründler (ed.), 100 Jahre Bezirkshauptmannschaften in Österreich, Wien, 1970, p. 9-17.

12. Ernst, Bruckmüller Nation Österreich. Kulturelles Bewußtsein und gesellschaftlich-politische Prozesse, Studien zu Politik und Verwaltung 4, Wien-Köln-Graz, Böhlau1984, p. 222 ; Ignaz Beidtel, Geschichte, vol. 1, 34, 69, 155f, 304f.

13. Pour la monarchie autrichienne : Hannelore Burger, «Passwesen [...]», op. cit., p. 3-87 ; Andrea Komlosy, Grenze [...], op.cit., p.87-95, p.297-300; plus généralement pour le processus d'identification voir Jane Caplan et John Torpey (eds.), Documenting Individual Identity: The Development of State Practises in the Modern World, Princeton, Princeton University Press, 1999; John Torpey, Origins of the Modern Passport System, Cambridge, Cambridge University Press, 1999.

14. Andrea Komlosy, Grenze [...], op. cit., p. 91. 
15. Konskriptionspatent vom 24.4.1781 - Handbuch aller unter der Regierung des Kaisers Joseph II. für die K.K. Erbländer ergangenen Verordnungen und Gesetze in einer systematischen Verbindung 1780-1789, 3, Wien, 1785, p. 37-95 ; Auswanderungspatent vom 18.8.1784 - Handbuch Joseph II., 6, Wien, 1786, p. 279-299 - compare A. Komlosy, Grenze [...], op. cit., p. 89.

16. Andrea Komlosy, Grenze [...], op. cit., p. 297-318.

17. Franz Tobias Herzog, Vollständige Sammlung der Gesetze über das Schubwesen im Kaiserthume Oesterreich, Wien, 1835 ; Franz Tobias Herzog, Sammlung der Gesetze über das politische Domizil im Kaiserthume Oesterreich, Wien, 1837 ; Harald Wendelin, «Schub und Heimatrecht ${ }^{\circ} »$, in Waltraud Heindl et Edith Saurer (ed), op. cit., p. 181-215.

18. Andrea Komlosy, Grenze [...], op. cit., p. 98 ; Mayrhofer Ernst et Pace Anton, Handbuch für den politischen Verwaltungsdienst in den im Reichsrathe vertretenen Königreichen und Ländern, 2, Wien, 1896, p. 974-1054.

19. Andrea Komlosy, Grenze [...], op. cit., p. 172 et annexes, tables B5, B7, B8.

20. Andrea Komlosy, Grenze [...], op. cit., p. 100 ; Gerhard Melinz et Susan Zimmermann, «:Über die Grenzen der Armenhilfe. Kommunale und staatliche Sozialpolitik in Wien und Budapest in der Doppelmonarchie ${ }^{\circ} »$, Materialien zur Arbeiterbewegung, 60, Wien-Zürich, 1991, p. 32-41 ; Wendelin, Schub, p. 231-339.

21. Gerhard Melinz et Susan Zimmermann, «Die aktive Stadt. Kommunale Politik zur Gestaltung städtischer Lebensbedingungen in Budapest, Prag und Wien (1867-1914) ${ }^{\circ}{ }^{\prime}$, in G. Melinz et S.Zimmermann (eds.), Wien-Prag-Budapest. Urbanisierung, Kommunal-politik, gesellschaftliche Konflikte (1867 - 1918), Wien, 1996, Promedia, p. 140-176.

22. Andrea Komlosy, Grenze [...], op. cit., p. 173.

23. Leopold Caro, «ํuswanderung und Auswanderungspolitik in Österreich»", Schriften des Vereins für Socialpolitik, 131, Leipzig, 1909; Karl Englisch, «Die oesterreichische Auswanderungsstatistik ${ }^{\circ} »$, in Statistische Monatsschrift NF 18 (1913), p.65-167; Heinz Faßmann,

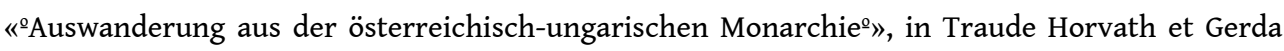
Neyer (ed.), Auswanderungen aus Österreich von der Mitte des 19. Jahrhunderts bis zur Gegenwart, WienKöln-Weimar, Böhlau, 1996, p. 33-56.

24. Komlosy, Grenze [...], op. cit., $190 \mathrm{f}$.

25. Cette distinction a survécu à la monarchie austro-hongroise; elle a servi de légitimation de «nationalité » par rapport aux déplacements et aux mouvements de réfugiés après la Deuxième Guerre mondiale.

\section{AUTEUR}

\section{ANDREA KOMLOSY}

Université de Vienne. Institut de sociologie 